\title{
Ethics in Neuroscience - current issues
}

\author{
Russell D'Souza \\ Director and Head, Asia-Pacific UNESCO Chair in Bioethics, Haifa. \\ Corresponding Author: Russell D’Souza \\ E-mail: russell.f.dsouza@gmail.com
}

The neurosciences and the cognitive sciences have shown rapid growth in research and knowledge development over the past 2 decades. With this increase in knowledge and research, there are multiple ethical issues that arise when considering the paradigms of neuroscience research. Neuroethics is a term coined which a subset of bioethics to understand and dissect the complex ethical questions that surround the new technologies ability to investigate and intervene in the brain. The aim of neuroethics is to shed light on areas such as the relationship between brain and mind and the nature of morality which makes up in fact the 'neuroscience of ethics' [1]. There have been increasing strides in psychiatric research that in turn affect neurosciences. Development of novel drugs that act on various neurotransmitters via different receptors in the brain and affect different neurochemical systems indirectly modulate the brain. There are far reaching ethical ramifications when such drugs are tested in clinical trials and alter the brain of healthy and diseased subjects while we ourselves do not fully understand the mechanisms that underlie its actions [2].

Some of the key areas under ethical debate in neuroscience are as follows -

\section{Neuroethics, Neuroenhancement and Wellness}

Nowadays a number of brain-based interventions are carried out with the aim of increasing attention, concentration, working memory and enhancing brain power. This is a realm of what is called neuro-enhancement. The aim is to maximize human potential and benefit many. Neuroenhancement involves costs that few patients can afford and thus there is no equality in the reach of the treatments. Is it right to modulate the brain in a manner that someone may do better in academics using neuroenhancement while others stay behind. Is it correct the modulate the brain and its physiology beyond normal to enhance outcomes and secure laurels in academics and sports. Does the neuro-enhancement have long term side effects that damage the brain and does it actually sound right to change neuroplasticity using machines. This is some of the dilemmas that neuro-enhancement and wellness poses.

\section{Brain imaging studies as evidence in a court of law}

Today we have huge enhancements in brain imaging and multi-modal techniques are available to help us study brain blood flow, metabolism, structure and function. These techniques can be used to elucidate the pathophysiology of various psychiatric disorders and help us determine brain correlates of these disorders as well as various day to day processes. Is it right to use neuroimaging data to understand the strengths and weaknesses of patients and then exploit the same in treatment. In the court of law is it ethical to present neuroimaging data which is actually personal data as evidence to save or sentence someone when implicated. Is neuroimaging data reliable and can be relied upon when data may fluctuate depending on the functional state and the situation that the brain is in. Is it right to use neuroimaging to enhance lie detection in a court of law. It is said that some of the imaging techniques may have upto $90 \%$ accuracy and one cannot change the brain like one may modulate the galvanic skin response in lie detection. Ethicists feel that using such data is a violation of personal privacy while forensic scientists feel otherwise. The debate is ongoing and a consensus is yet to be reached [4-5]. 


\section{Neuroimaging to enhance recruitment and organizations}

Neuroimaging can help scientists tap ones thought and behavior patterns as well as the capacity for judgement. Should neuroimaging be used to judge such behaviors, rather than just pure observations and interviews. Should one use neuroimaging to screen for high posts and jobs so that the right people are recruited. Organizational neuroscience has great promise for advancing organizational research and practice in daily work. This field is developing rapidly and is plagued by technological and methodological challenges that have implications when conducting or interpreting neuroscience research in organizations and in the context of organizational behavior. Can neuroscientific research be made meaningful in organizations or is neuroscience as just another management fad. Would a person lose a job opportunity if he does not consent for imaging to get the job. Is it right to use extremely private biological data for organizational wellbeing and to judge differences between two human beings and what happens between them [6].

\section{Neurotalk}

Neurotalk is the concept on how neuroscience is conveyed to the common man. There are many books in pop psychology that look at the brain, how the brain changes itself, how to use your brain fully and how to mould your brain and so on. It is imperative that readers are conveyed the truth about neuroscience. Research is a double edged sword and semantics may be used to misconstrue research findings and present a completely different picture to the audience that reads. It is therefore essential that neuroscientists are ethical in the promises that neurosciences make and how neuroscience research is conveyed to the common man. Myths related to brain called neuromyths need to be clarified and facts about the brain need to be explained. There is no need to glamorize neurology and psychiatry as professions and rather the humane angle of these sciences must be emphasized. There is also a need to understand that by writing about cases that are extraordinary and some patients may be misguided that everyone has a miraculous recovery especially in disorders where recovery may be difficult [8].

\section{Brain privacy and Cognitive Liberty}

Functional neuroimaging has been used in research to study many psychological traits including personality, aggression, anxiety and intelligence. While we have made progress in identifying the neural correlates of many traits and the individual differences between them this has raised ethical concerns about privacy. Brain privacy is the concept where one's brain and the data derived from neuroimaging need to be confidential. In this era of neuroimaging where brainotyping is done for must patients one must be careful when addressing this issue in studies that involve meta-analysis. Every human being is different and we must be very careful to address the fact that it is wrong to pool data and come with consensus about the human race [9].

With modern imaging and genome sequencing, microprocessors are becoming faster and smaller and the human DNA sequence has been developed. We, as a society, must acknowledge the fundamental human right to cognitive liberty and must delineate its boundaries. Cognitive liberty encroachments may be in various ways where new technologies such as biogenetic modification, human-computer interfacing, brain-scanning, nanotechnology, neural-networking are giving us insights into how we were born and how our brains have evolved. The trend of technology is to overcome the limitations of the human body and we must not forget that we as humans have developed technology and we are humane first. As mentioned by researchers, "What are the implications for mental autonomy when wearable computers become wet-wired to our own minds and memory is augmented by a high-speed wireless connection to the Web?". Hence there is a need for brain privacy and cognitive liberty [10].

\section{Brain Fingerprinting and Brain Reading}

Studies have sought neuroimaging correlates of various dimensions of personality such as extraversion and neuroticism. One of the most sought-after uses of 'brain reading' is the detection of deception. Post the world trade centre attacks, there is renewed interest in lie detection for security purposes and to screen individuals for their attitudes and for traditional forensic purposes. There is a company Brain Fingerprinting Laboratories which is researching and marketing a 
computer system that uses scalp-recorded ERPs to detect so-called 'guilty knowledge', such as familiarity with certain people, objects or scenes. Research seeking more neuroanatomically specific measures of deception using fMRI is underway. There are huge ethical dimensions as the chance that neuroimaging findings may be wrong is also likely. Imaging may provide the most sensitive and specific measures available of psychological processes but there is a risk that juries, judges, parole boards, the immigration service and so on will weight such measures too heavily in their decision making and thus affect the human trust that exists in such systems [11].

\section{Conclusions}

Neuroethics refers to two related fields of study: what the philosopher Adina Roskies has called the ethics of neuroscience, and the neuroscience of ethics. The ethics of neuroscience comprises the bulk of work in neuroethics and concerns the ethical, legal and social impact of neuroscience, including the ways in which neurotechnology can be used to predict or alter human behavior and the implications of our mechanistic understanding of brain function for society.

The neuroscience of ethics is how the brain processes ethical dilemmas and the neural correlates used in this process. The present editorial looked at some of burning issues in the ethics of neuroscience and presented some of the bog questions in neuroethics that haunt us today. There is need for clinicians, patients, caregivers, neuroscientists and students to be aware of these ethical dilemmas as they venture into the field of neuroscience.

Neuroethics poses interesting crossroads for psychiatric research of the future and ranges from whether this research is needs to the long term ramifications of these research projects and how these research projects can be used for the greater good of society and mankind in general. The chapter gave the reader a bird's eye view of these issues and shall enable the reader to think deeper and further into these dilemmas.

\section{REFERENCES}

1. Levy N. Neuroethics: Challenges for the 21st century. Cambridge University Press 2007.

2. Farah MJ. Neuroethics and the problem of other minds: Implications of neuroscience for the moral status of brain-damaged patients and nonhuman animals. Neuroethics 2008;1(1):9-18.

3. Heinz A, Kipke R, Heimann H, Wiesing U. Cognitive neuroenhancement: false assumptions in the ethical debate. J Med Ethics 2012;38(6):372-5.

4. Meegan DV. Neuroimaging techniques for memory detection: scientific, ethical, and legal issues. Am J Bioethics 2008;8(1):9-20.

5. Canli T, Amin Z. Neuroimaging of emotion and personality: Scientific evidence and ethical considerations. Brain Cogn 2002;50(3):414-31.

6. Lee N, Chamberlain L. Neuroimaging and psychophysiological measurement in organizational research. Ann NY Acad Sci 2007;1118(1):18-42.

7. Illes J, Moser MA, McCormick JB, Racine E, Blakeslee S, Caplan A, Hayden EC, Ingram J, Lohwater T, McKnight P, Nicholson C. Neurotalk: improving the communication of neuroscience research. Nat Rev Neurosci 2010;11(1):61-8.

8. Farah MJ. Neuroethics and the problem of other minds: Implications of neuroscience for the moral status of brain-damaged patients and nonhuman animals. Neuroethics 2008;1(1):9-18.

9. Fuchs T. Ethical issues in neuroscience. Curr Opin Psychiatry 2006;19(6):600-7.

10. Sententia W. Neuroethical considerations: cognitive liberty and converging technologies for improving human cognition. Ann NY Acad Sci 2004;1013(1):221-8.

11. Tennison MN, Moreno JD. Neuroscience, ethics, and national security: the state of the art. PLoS Biol 2012;10(3):e1001289.

$$
\begin{aligned}
& \text { Acknowledgements - Nil } \\
& \text { Source of Funding - Nil } \\
& \text { Conflict of Interest - Nil }
\end{aligned}
$$

